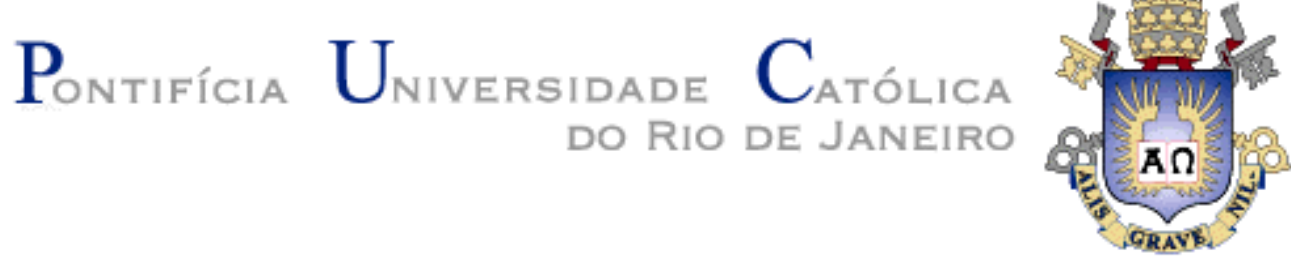

Ticiana Mascarenhas Galeão Neves

\title{
Principais fatores de mercado que determinam a decisão sobre creches na cidade do Rio de Janeiro
}

Dissertação de Mestrado

Dissertação apresentada como requisito parcial para obtenção do grau de Mestre pelo Programa de PósGraduação em Administração de Empresas do Departamento de Administração do Centro de Ciências Sociais da PUC-Rio

Orientador: Prof. Jorge Ferreira da Silva

Rio de Janeiro

Abril de 2009 


\title{
Ticiana Mascarenhas Galeão Neves
}

\author{
Principais fatores de mercado que determinam a \\ decisão sobre creches na cidade do Rio de Janeiro
}

Dissertação apresentada como requisito parcial para obtenção do grau de Mestre pelo Programa de Pós-Graduação em Administração de Empresas do Departamento de Administração do Centro de Ciências Sociais da PUC-Rio. Aprovada pela Comissão Examinadora abaixo assinada

Prof. Jorge Ferreira da Silva Orientador Departamento de Administração - PUC-Rio

Prof ${ }^{a}$. Marie Agnes Chauvel Departamento de Administração - PUC-Rio

Profa. Leticia Moreira Casotti Universidade Federal do Rio de Janeiro

Prof. Nizar Messari Vice-Decano de Pós-Graduação do CCS 
Todos os direitos reservados. É proibida a reprodução total ou parcial do trabalho sem a autorização da universidade, da autora e do orientador.

\section{Ticiana Mascarenhas Galeão Neves}

Graduou-se em Engenharia Elétrica com ênfase em Eletrônica (Universidade Federal do Rio de Janeiro) em 1997. No âmbito profissional atuou como consultora de sistemas na PriceWaterhouseCoopers e em diversas áreas na Xerox do Brasil.

Ficha Catalográfica

\section{Neves, Ticiana Mascarenhas Galeão}

Principais fatores de mercado que determinam a decisão sobre creches na cidade do Rio de Janeiro / Ticiana Mascarenhas Galeão Neves ; orientador: Jorge Ferreira da Silva. - 2009.

97 f. : il. ; $30 \mathrm{~cm}$

Dissertação (Mestrado em Administração)Pontifícia Universidade Católica do Rio de Janeiro, Rio de Janeiro, 2009.

Inclui bibliografia

1. Administração - Teses. 2. Desenvolvimento de produtos. 3. Marketing de serviços. 4. Análise conjunta. 5. Segmentação de mercado. 6. Creche. I. Silva, Jorge Ferreira da. II. Pontifícia Universidade Católica do Rio de Janeiro. Departamento de Administração. III. Título.

CDD: 658 
Este trabalho é dedicado a minha filha Liz, inspiração para o tema, de quem roubei momentos preciosos na confecção desta pesquisa. 


\section{Agradecimentos}

Ao meu marido, Inon, sem cujo incondicional apoio teria sido impossível chegar até aqui.

Aos meus pais, por me ensinarem que o conhecimento é bem mais valioso que existe e o único que jamais pode ser roubado.

Às amigas, Renata Azevedo e Renata Coutinho, pela ajuda nos momentos difíceis.

A todos que colaboraram, me auxiliando na coleta dos dados ou participando das entrevistas, grupo de foco ou preenchimento dos questionários.

Ao meu orientador, Prof. Jorge Ferreira, pelo suporte para a realização do trabalho. 


\section{Resumo}

Neves, Ticiana Mascarenhas Galeão; Silva, Jorge Ferreira. Principais fatores de mercado que determinam a decisão sobre creches na cidade do Rio de Janeiro. Rio de Janeiro, 2009. 97p. Dissertação de Mestrado - Departamento de Administração, Pontifícia Universidade Católica do Rio de Janeiro.

A primeira infância é fundamental para o desenvolvimento físico e psíquico dos seres humanos, influenciando-os pelo resto da vida. Assim sendo, a oferta de serviços de creche adequados, calcados no correto mapeamento dos desejos e necessidades dos consumidores, é de extrema importância. Partindo desta motivação, este trabalho objetiva analisar as características relevantes para os pais no processo de seleção de creches. A dissertação é baseada na metodologia da análise conjunta e seu desenvolvimento é composto de duas etapas: i. Revisão da literatura, entrevistas em profundidade, grupo de foco e definição dos estímulos a serem avaliados pelos respondentes; ii. Coleta de dados, cálculo das importâncias relativas dos atributos, cálculo das utilidades dos níveis propostos e análise dos resultados. A análise dos resultados é feita considerando-se não somente o total da amostra como também segmentos definidos a priori de acordo com dados sócio-econômicos levantados na coleta de dados. O resultado da análise conjunta para o total da amostra revela os atributos localização, projeto pedagógico e grau de capacitação dos profissionais como sendo os de maior importância relativa. Este resultado evidencia que, muito além da praticidade de creches que sejam guardiãs de seus filhos, os pais buscam também uma instituição que propicie o aprendizado e a socialização. Na segmentação, os resultados por região de residência, faixa etária das crianças e faixa de renda familiar possibilitaram a definição de estratégias específicas para cada segmento.

\section{Palavras-chave}

Desenvolvimento de produtos; marketing de serviços; análise conjunta; segmentação de mercado; creche. 


\section{Abstract}

Neves, Ticiana Mascarenhas Galeão; Silva, Jorge Ferreira (Advisor). Main Market Determinants of Child Care Services in Rio de Janeiro. Rio de Janeiro, 2009. 97p. MSc. Dissertation - Departamento de Administração, Pontifícia Universidade Católica do Rio de Janeiro.

The first childhood is fundamental for the physical and psychological development of human beings, influencing them for the rest of their lives. In this way, offering adequate child care services, based in a correct mapping of customers' necessities is extremely important. Derived from this motivation, this work aims to analyze the relevant characteristics for parents in the child care services selection process. The dissertation is based on conjoint analysis methodology and is developed in two parts: i. Literature revision, interviews, focus groups and definition of the stimulus to be evaluated by the respondents; ii. Data collection, calculation of the relative importance of the attributes, calculation of the utilities of each suggested level and analyses of the results. The analysis is done considering not only the total sample but also segments defined according to socio-economical data gathered as part of the data collection process. The result of the conjoint analysis for the overall sample reveals that location, teaching methodology and professional knowledge and skills received the greatest relative importance. It shows that, far beyond a practical child care service that can look after their children, parents are aiming for an institution that leverages development, socialization and learning. When segmenting the data, analyses by neighborhood, children's age and familiar income enabled the definition of strategies for each specific segment.

\section{Keywords}

Product development; services marketing; conjoint analysis; market segmentation; child care. 


\section{Sumário}

1 Descrição do Problema 12

1.1. Introdução 12

$\begin{array}{ll}\text { 1.2. Objetivos da Pesquisa } & 14\end{array}$

1.3. Relevância do Estudo 15

1.4. Delimitação do Estudo 16

2 Referencial Teórico 17

$\begin{array}{ll}\text { 2.1. Marketing de Serviços } & 17\end{array}$

2.2. Desenvolvimento de Produtos 22

2.3. Análise Conjunta 28

2.4. Segmentação de Mercado $\quad 37$

3 Metodologia de Pesquisa 41

3.1. Tipo de Pesquisa 41

3.2. Universo e Amostra 41

3.3. Coleta de Dados 44

3.4. Tratamento dos Dados 45

3.5. Limitações do Método 46

3.6. Seleção de Atributos e Níveis 47

3.7. Seleção das Opções de Compra 56

$\begin{array}{ll}\text { 3.8. Pesquisa de Campo } & 59\end{array}$

4 Resultados $\quad 61$

4.1. Resultados Gerais da Amostra 61

4.2. Análise dos Resultados por Segmentos 66

5 Conclusão 73

5.1. Sugestão para Pesquisas Futuras 76

6 Referências Bibliográficas $\quad 78$ 
7 Anexos

7.1. Roteiro para Entrevista em Profundidade 82

7.2. Questionário de pré-teste 84

7.3. Questionário Final 92

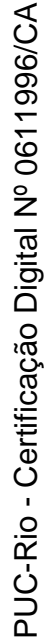




\section{Lista de Tabelas}

Tabela 1 - Priorização dos atributos sugeridos 52

Tabela 2 - Priorização dos atributos adicionados 53

Tabela 3 - Seleção dos atributos $\quad 53$

Tabela 4 - Descrição dos atributos e níveis 56

Tabela 5 - Descrição dos atributos e níveis - Seleção Final 58

Tabela 6 - Seleção Final das Opções de Compra 59

Tabela 7 - Ordem de Preferência dos Cartões Apresentados 62

Tabela 8 - Resultados para o Total da Amostra 64 


\section{Lista de Figuras}

Figura 1. Espectro da Tangibilidade $\quad 20$

Figura 2. Processo de Desenvolvimento de Produto 26

Figura 3. Passo a Passo da Análise Conjunta 30

Figura 4. Gráfico das Funções de Valores Parciais 35

Figura 5. Distribuição da amostra por gênero 42

Figura 6. Distribuição da amostra entre pais que vivem juntos e pais que vivem separados $\quad 42$

Figura 7. Distribuição da amostra por região 43

Figura 8. Distribuição da amostra por faixa etária 43

Figura 9. Distribuição da amostra por faixa etária com a qual os filhos entraram na creche $\quad 44$

Figura 10. Distribuição da amostra por renda familiar 44

Figura 11. Importância relativa dos atributos por gênero 67

Figura 12. Importância relativa dos atributos - Pais que $\begin{array}{ll}\text { vivem juntos } x \text { Pais que vivem separados } & 68\end{array}$

Figura 13. Importância relativa dos atributos por região 69

Figura 14. Importância relativa dos atributos por faixa etária do respondente $\quad 71$

Figura 15. Importância relativa dos atributos por faixa etária da criança $\quad 71$

Figura 16. Importância relativa dos atributos por renda familiar 